\title{
Efficacy of colistin in multidrug-resistant neonatal sepsis: experience from a tertiary care center in Karachi, Pakistan
}

\author{
Gul Ambreen (1) , 'Muhammad Sohail Salat, ${ }^{2}$ Kashif Hussain, ${ }^{1}$ Syed Shamim Raza, \\ Umer Ali, ${ }^{1}$ Iqbal Azam, ${ }^{3}$ Junaid Iqbal, ${ }^{2}$ Zafar Fatmi $^{3}$
}

${ }^{1}$ Pharmacy, Aga Khan University Hospital, Karachi, Pakistan ${ }^{2}$ Pediatric and Child Health, Aga Khan University, Karachi, Pakistan

${ }^{3}$ Community Health Sciences, Aga Khan University, Karachi, Pakistan

\section{Correspondence to}

Dr Gul Ambreen, Pharmacy, Aga Khan University, Karachi 74800, Pakistan; gul.ambreen@aku.edu

Received 15 August 2019 Revised 26 February 2020 Accepted 27 February 2020 Published Online First 20 March 2020
Check for updates

(C) Author(s) (or their employer(s)) 2020. No commercial re-use. See rights and permissions. Published by BMJ.

To cite: Ambreen $\mathrm{G}$, Salat MS, Hussain K, et al. Arch Dis Child

2020;105:830-836.

\section{ABSTRACT}

Objective Infections with multidrug-resistant organisms (MDROs) such as Gram-negative bacteria have high morbidity and mortality with limited treatment options. Colistin, an antibiotic active against MDRO, was rarely used due to frequent adverse effects, but its use has now been recommended among adults. In this study, we determined the efficacy of colistin for the treatment of sepsis in neonates.

Design/setting/patients/outcomes We conducted a retrospective record review of all neonates admitted to the neonatal intensive care unit of Aga Khan University Hospital, Karachi, Pakistan, between June 2015 and June 2018, who had sepsis and received colistin by intravenous, inhalation and/or intrathecal routes. Predictors of colistin efficacy, for neonatal survival and microbial clearance, were assessed using multiple logistic regression.

Results 153 neonates received colistin; 120 had culture-proven sepsis; and 93 had MDR-GNB (84 colistin-sensitive). 111 (72.5\%) neonates survived and were discharged from hospital; $82.6 \%$ had microbial clearance. Neonates with colistin-sensitive bacteria (adjusted OR (AOR)=3.2, 95\% Cl 2.8 to 4.0), and those in which colistin therapy started early $(\mathrm{AOR}=7.2,95 \% \mathrm{Cl}$ 3.5 to 13.6) were more likely to survive. Neonates with increased gestational age ( $A O R=1.9,95 \% \mathrm{Cl} 1.5$ to 3.0), higher weight ( $A O R=5.4,95 \% \mathrm{Cl} 3.3$ to 11.8$)$ and later onset of sepsis ( $A O R=4.3,95 \% \mathrm{Cl} 2.0$ to 9.0 ) had higher survival. Adverse events included nephrotoxicity in 5.2\%; $13.7 \%$ developed seizures and $18.3 \%$ had electrolyte imbalance.

Conclusions Colistin therapy was associated with survival among neonates suffering from MDR-GNB sepsis. The frequency of side effects was moderate.

\section{BACKGROUND}

Infection with multidrug-resistant Gram-negativebacteria (MDR-GNB) is associated with high morbidity and mortality, ${ }^{12}$ and often these infections are nosocomial. The incidence of neonatal nosocomial infections has been reported between $7 \%$ and $24 \%$ in South Asia. ${ }^{3}$ Acinetobacter baumannii, Klebsiella pneumoniae, Pseudomonas aeruginosa and Echerichia coli are the most frequent Gram-negative-bacteria (GNB) in South Asia with a high rate of multidrug resistance. ${ }^{45}$ In one study, $89 \%$ of Acinetobacter species were MDR. ${ }^{4}$ Furthermore, carbapenem-resistant organisms (CROs) are among multidrug-resistant organisms (MDROs)

\section{What is already known on this topic?}

- Multidrug-resistant Gram-negative bacteria (MDR-GNB) are a big challenge in the neonatal population in Pakistan.

- The number of MDR-GNB infections is increasing in neonates.

- Colistin is considered a therapeutic option after the failure of carbapenem use.

\section{What this study adds?}

- Predictors of response to colistin and significant association with neonatal survival with earlier microbial clearance.

- Starting colistin within 1-2 hours of the onset of severe sepsis or septic shock may improve the survival of neonates with colistin-sensitive MDR-GNB infections.

- Intrathecal colistin may improve the survival of neonates with MDR-GNB meningitis and ventriculitis.

which pose the greatest challenge with mortality ranging from $12 \%$ to $50 \%$ among neonates. ${ }^{5-7}$

Colistimethate sodium (CMS) is an inactive prodrug administered parenterally. It is then hydrolysed into its active metabolite colistin, which is a potent antimicrobial agent; CMS use was abandoned earlier due to frequent side effects. However, more recently, CMS has been recommended for adults with MDR-GNB infections. ${ }^{8} 9$ CMS treatment is particularly recommended when carbapenem resistance is reported. Cefiderocol is another therapeutic agent effective against MDRO like CROs. ${ }^{10} 11$ However, cefiderocol and similar agents are expensive with limited availability in low-income and middle-income countries (LMICs). Colistin is available in LMICs and is used for most of the susceptible CROs. ${ }^{12}$ Colistin can cause a high rate of neurotoxicity and nephrotoxicity if administered in high doses intramuscularly, although toxicities are negligible when given intravenously. ${ }^{13}$ Colistin has been used widely among adults with cancer, burns and in intensive care units. ${ }^{14} 15$ There are very few studies describing colistin use among children and neonates. ${ }^{9} 16$ Determining the safety of colistin use among neonates admitted to ICU 
is difficult due to the presence of other contributing comorbid conditions, including prematurity and use of other antibiotics. Thus, the safety and efficacy of colistin among neonates remain unclear, particularly in LMIC settings. ${ }^{17} 18$

The purpose of this study was to evaluate the use of colistin in a neonatal intensive care unit (NICU) setting in Pakistan. We investigated the efficacy and safety of colistin in the neonatal population considering coexisting clinical conditions and concomitant medicinal use.

\section{METHOD}

Study design, participants' eligibility and data sources

This was a retrospective review of medical records from a tertiary care hospital in Karachi, Pakistan. Infants admitted to NICU and the step-down unit, between June 2015 and June 2018 , receiving colistin by any route (intravenous, nebuliser or intrathecal) for sepsis, were included in the study. Patients with seizures, acute kidney injury (AKI) or congenital abnormalities of the urinary tract were excluded.

Information was extracted from hospital electronic records. This included information on clinical characteristics and route, dose and duration of colistin therapy. Laboratory parameters like serum creatinine, blood urea nitrogen, serum electrolytes at baseline; days 1, 3 and 7, and then weekly until the end of colistin therapy were also recorded. We recorded concomitant use of other nephrotoxic drugs/antibiotics (within 7 days of colistin therapy) and the occurrence of seizures. In addition, data for culture and sensitivity, organism eradication period and survival/death was also retrieved.

\section{Definitions}

Clinical sepsis and nosocomial infection were defined as per standard definitions from the Centers for Disease Control and Prevention. ${ }^{19}$ Neonates who had sepsis associated with any organ dysfunction, hypotension or on vasopressors were classified as having septic shock. ${ }^{19}$ Neonate with a clinical presentation of septicaemia, meningitis or pneumonia, along with pathogen isolation in control cultures of blood, cerebrospinal fluid (CSF), endotracheal aspirate or urine were defined as culture-positive sepsis. Treatment failure was considered when the same condition persisted or got worse over the period of $48-72$ hours or new infection occurred or signs of worsening on radiology. ${ }^{20}$

We used the modified Kidney Disease Improving Global Outcomes definition for AKI. ${ }^{21}$ AKI assessed by changes in serum level creatinine (before, during and after the treatment) and defined as an increase in serum creatinine (at least $0.3 \mathrm{mg} / \mathrm{dL}$ within 48 hours or $150 \%-200 \%$ increase from baseline trough value). ${ }^{21}$ Neurotoxicity was defined as an episode of seizure or change in consciousness level at any time during colistin therapy. We defined microbiological response as no bacterial growth in cultures of CSF, blood or urine, at least 72 hours after starting colistin therapy. Cultures were collected and evaluated every 48 hours until microbial clearance. MDRO was defined as an isolate non-susceptible to at least one agent of $\geq 3$ antimicrobial categories. ${ }^{22}$ Hypomagnesaemia was defined as serum magnesium concentration of $<1.6 \mathrm{mg} / \mathrm{dL}^{23}$ and hypokalaemia as serum potassium concentrations of $<3.5 \mathrm{mEq} / \mathrm{L} .{ }^{24}$ The efficacy of colistin as an outcome measure was evaluated based on the microbiological clearance of cultures and patient survival with colistin therapy.

\section{Microbiological methods}

Baseline and subsequent cultures during therapy were taken from venous blood, endotracheal aspirate, CSF, urine, wound/tissue/ pus or central venous catheter. These culture samples were inoculated on routine culture media (blood/chocolate/MacConkey agar). Pathogens were identified through Vitek-2 compact system and biochemical tests. Colistin susceptibility of isolated pathogens was confirmed by performing a broth microdilution test. Isolated pathogens with minimum inhibitory concentration (MIC) of $\leq 2 \mathrm{mg} / \mathrm{dL}$ were documented as colistin-sensitive using Clinical Laboratory Standards Institute (CLSI) guidelines. ${ }^{25}$

Institutional antibiotic guidelines were followed for the treatment of neonatal sepsis. First-line therapy included intravenous ampicillin and gentamicin or cefotaxime and amikacin. Secondline therapy was carbapenem with vancomycin. Colistin was reserved as a third-line antibiotic, mostly administered with meropenem. Colistin therapy was started; 1 . after culture sensitivity reports; 2 . as antimicrobial escalation for failure of first and second-line antibiotics; 3 . in cases of severe sepsis/septic shock within 1-2 hours empirically with carbapenem based on our NICU antimicrobial susceptibility patterns. ${ }^{45}{ }^{26}$ Since colistin is considered a controlled medication, approval from an infectious disease consultant was needed in non-culture proven sepsis when therapy continued for more than 72 hours.

Colistin (base) was administered intravenously at a dose of $2.5-5.0 \mathrm{mg}$ (75 000-150 000 units)/ $\mathrm{kg} /$ day in two to four divided doses, $4 \mathrm{mg} / \mathrm{kg} /$ dose twice daily via nebulisation and 0.16 $0.24 \mathrm{mg} / \mathrm{kg}$ as a single daily dose via intrathecal/intraventricular route. Doses were adjusted in patients with reduced renal function. ${ }^{27-29}$ All intravenous and intrathecal doses were dispensed in prefilled ready to administer form in normal saline and infused over $30 \mathrm{~min}$.

\section{Outcome measures and statistical analysis}

Primary outcome measures were microbiological clearance and patient survival during treatment. Colistin-associated adverse effects, such as nephrotoxicity, neurotoxicity and electrolyte imbalance, were evaluated as secondary outcomes.

Data were analysed using IBM SPSS V.20. Descriptive statistics were provided according to those neonates who survived and died. At the univariate level, each independent variable was analysed to see the association of survival and microbial clearance using binary logistic regression. Those variables with significance of $\leq 0.20$ or biologically plausible (parameters related to antenatal care and neonatal clinical parameters) at univariate level were considered for multivariable analysis.

\section{RESULTS}

A total of 153 patients with sepsis who underwent colistin therapy were included in this study (see table 1 ). Most were male $(n=102)$, preterm $(n=118)$ and low birth weight $(n=110)$. Bacteraemia was the most common diagnosis $(n=108)$ followed by pneumonia $(n=34)$ and meningitis $(n=15) ; 120$ were cultureproven cases, while the rest were diagnosed on clinical grounds K. pneumonia and A. baumannii were the most frequent organisms isolated. Multiple comorbid conditions were present among neonates. Nearly three quarters $(72.5 \%)$ of neonates survived.

Colistin-sensitive organisms were found in 64 surviving and 20 neonates who died, while colistin-resistant organisms were found in 1 and 8 neonates who survived and died respectively. Concomitant use of carbapenem was $91.0 \%$ in those who survived and $90.5 \%$ in those who died. There was no difference in the mean duration of colistin therapy in those who survived 


\section{Original research}

Table 1 Demographic, hospital management and disease status of neonates with sepsis administered colistin therapy who survived or died at a tertiary care hospital in Karachi $(\mathrm{N}=153)$

\begin{tabular}{|c|c|c|}
\hline & Survived & Died \\
\hline Variables & $(n=111)(\%)$ & $(n=42)(\%)$ \\
\hline \multicolumn{3}{|l|}{ Demographic/birth history } \\
\hline Male & $79(71.2)$ & $23(54.8)$ \\
\hline Delivery by caesarean section & $49(44.1)$ & $27(57.1)$ \\
\hline Premature rupture of membranes ( $>18$ hours before delivery) & $15(13.5)$ & $9(21.4)$ \\
\hline \multicolumn{3}{|l|}{ Hospital stay/management of patient } \\
\hline \multicolumn{3}{|l|}{ Comorbid conditions/illness } \\
\hline Respiratory* & $65(58.6)$ & $31(73.8)$ \\
\hline Neurologicalt & $37(33.3)$ & $10(23.8)$ \\
\hline Gastrointestinal‡ & $21(18.9)$ & 7 (16.7) \\
\hline Cardiac§ & $15(13.5)$ & $7(16.7)$ \\
\hline Metabolic/endocrinef & $1(0.9)$ & $4(9.5)$ \\
\hline \multicolumn{3}{|l|}{ Diagnosis/site of infection } \\
\hline Bacteraemia & $84(75.7)$ & $24(57.1)$ \\
\hline Meningitis & $6(5.4)$ & $9(21.4)$ \\
\hline Pneumonia & $25(22.5)$ & $9(21.4)$ \\
\hline \multicolumn{3}{|l|}{ Isolated microbes and their sensitivity } \\
\hline \multicolumn{3}{|l|}{ Isolated microorganisms } \\
\hline Klebsiella pneumonia & $34(30.6)$ & $8(19)$ \\
\hline Acinetobacter baumannii & $33(29.7)$ & $10(23.8)$ \\
\hline Enterobacter species & $10(9.0)$ & $2(4.8)$ \\
\hline Pseudomonas aeruginosa & $9(8.1)$ & $4(9.5)$ \\
\hline Escherichia coli & $4(3.6)$ & $2(4.8)$ \\
\hline Elizabethkingia meningoseptica & $2(1.8)$ & 0 \\
\hline \multicolumn{3}{|l|}{ Site of positive culture } \\
\hline Blood & $50(45.0)$ & $20(47.6)$ \\
\hline Blood (+tracheal/CSF/urine/tissue) & $28(25.2)$ & $6(14.3)$ \\
\hline Other than blood & $4(9.9)$ & $5(11.9)$ \\
\hline \multicolumn{3}{|l|}{ Micro-organism sensitivity } \\
\hline Culture-proven sepsis & $90(81.1)$ & $30(71.4)$ \\
\hline Colistin-resistant MDR-GNB & $8(7.2)$ & $1(2.4)$ \\
\hline Colistin-sensitive MDR-GNB & $64(57.7)$ & $20(47.6)$ \\
\hline Carbapenem-sensitive/no susceptibility check for colistin & $18(16.2)$ & $9(21.4)$ \\
\hline No growth/clinical sepsis & $21(18.9)$ & $12(28.6)$ \\
\hline \multicolumn{3}{|l|}{ Medication/management } \\
\hline \multicolumn{3}{|l|}{ Concomitant medications (antibiotic/nephrotoxic agent)** } \\
\hline Carbapenem & $101(91.0)$ & $38(90.5)$ \\
\hline Aminoglycosides & $87(78.4)$ & $33(78.6)$ \\
\hline Cephalosporin (third generation) & $86(77.5)$ & $33(78.3)$ \\
\hline Vancomycin & $81(73.0)$ & $32(76.2)$ \\
\hline Diuretics (more than three doses) & $67(60.4)$ & $27(64.3)$ \\
\hline Amphotericin B & $16(14.4)$ & 7 (16.7) \\
\hline Piperacillin/tazobactam & $4(3.6)$ & $6(14.3)$ \\
\hline Ibuprofen & $5(4.5)$ & $3(7.1)$ \\
\hline \multicolumn{3}{|l|}{ Route of administration of colistin } \\
\hline Intravenous only & $85(76.6)$ & $35(83.3)$ \\
\hline Aerosolised only & $1(0.9)$ & $2(4.8)$ \\
\hline Intravenous and aerosolised & $19(17.1)$ & $4(9.5)$ \\
\hline Intravenous and intrathecal & $5(4.5)$ & 0 \\
\hline Intrathecal only & $1(0.9)$ & $1(2.4)$ \\
\hline \multicolumn{3}{|l|}{ Mean duration of colistin therapy (days) $+\dagger$} \\
\hline Intravenous & $8.2(6.5)$ & $8.1(6.6)$ \\
\hline Aerosolised & $5.8(3.6)$ & $3.3(1.5)$ \\
\hline Intrathecal & $5.3(1.9)$ & $6.2(1.8)$ \\
\hline
\end{tabular}


Table 1 Continued

\begin{tabular}{|c|c|c|}
\hline & Survived & Died \\
\hline Variables & $(n=111)(\%)$ & $(n=42)(\%)$ \\
\hline BUN (mg/dL) & $20.4(1.3)$ & $22.5(1.6)$ \\
\hline Creatinine (mg/dL) & $0.62(0.31)$ & $0.58(0.25)$ \\
\hline Magnesium (mg/dL) & $1.8(0.4)$ & $1.3(0.3)$ \\
\hline Potassium (mEq/L) & $3.4(0.58)$ & $3.0(0.49)$ \\
\hline Calcium (mg/dL) & $8.7(0.6)$ & $7.6(0.8)$ \\
\hline Sodium (mEq/L) & $141(3.6)$ & $138(2.1)$ \\
\hline \multicolumn{3}{|l|}{ Adverse effects experienced during colistin therapy } \\
\hline Needed additional electrolytes supplementation & $18(16.2)$ & $10(23.8)$ \\
\hline Seizures & $14(12.6)$ & $7(16.7)$ \\
\hline Acute kidney injury & $7(6.3)$ & $1(2.4)$ \\
\hline Mean length of NICU stay (days) $+\dagger$ & $12.5(10.8)$ & $15.3(9.8)$ \\
\hline Mean length of hospital stay (days) $\dagger \dagger$ & $20.7(13.4)$ & $16.9(9.2)$ \\
\hline
\end{tabular}

* Respiratory illness: bronchopulmonary dysplasia, bronchiolitis, transient tachypnea of the newborn, respiratory distress syndrome, persistent pulmonary hypertension of the newborn and meconium aspiration syndrome.

tNeurological illnesses: perinatal asphyxia, meningomyelocele, Down's syndrome, intraventricular haemorrhage, patent ductus arteriosus and presence of external ventricular drain/ventriculoperitoneal shunt.

¥Gastrointestinal illnesses: necrotising enterocolitis, oesophageal atresia and duodenal oesophageal atresia.

$\S$ Cardiac illnesses: acyanotic congenital heart disease and cardiac surgery.

ףMetabolic/endocrine illnesses: infant of a diabetic mother, hypoglycaemia, maple syrup urine disease and inborn errors of metabolism.

${ }^{*}$ Used within 1 week before and/or during colistin therapy.

††Mean \pm SD.

BUN, blood urea nitrogen; CSF, cerebrospinal fluid; MDR-GNB, multidrug-resistant Gram-negative bacteria; NICU, neonatal intensive care unit.

versus those who died (table 1 ). The median life span of those neonates who died was 23 days (range 5-45 days). Adverse events associated with colistin use included AKI $(n=8,5.2 \%)$, seizures $(n=21,13.7 \%)$ and electrolyte imbalance $(n=28,18.3 \%)$.

Table 2 describes the adjusted OR (AOR) for factors associated with survival among neonates with colistin use. Neonates with a positive culture and colistin-sensitive organisms were more likely to survive ( $\mathrm{AOR}=3.2,95 \% \mathrm{CI} 2.8$ to 4.0$)$. Early initiation of colistin after the onset of severe sepsis/septic shock $(\mathrm{AOR}=7.2$, $95 \%$ CI 3.5 to 13.6 ) led to a higher survival rate. Microbial clearance (AOR $=5.2,95 \% \mathrm{CI} 2.2$ to 13.2 ) was more likely to be associated with a higher survival rate. Other factors associated with improved survival included later onset of sepsis in the hospital, increased gestational age (GA), higher birth weight and absence of deteriorating signs.

Table 3 describes the AOR for factors associated with microbial clearance in neonates with colistin use. Colistin administered within 1-2 hours of the onset of sepsis/septic shock had much higher microbial clearance $(\mathrm{AOR}=32.9,95 \% \mathrm{CI} 15.4$ to 58.5$)$. Simultaneous use of carbapenem had no advantage for microbial clearance. Neonates with colistin-sensitive organisms were more likely to have microbial clearance $(\mathrm{AOR}=2.1,95 \% \mathrm{CI} 1.0$ to 3.7). Later onset of sepsis in the hospital (AOR $=2.0,95 \% \mathrm{CI}$ 1.0 to 1.5$)$ and higher birth weight were associated with higher

Table 2 Factors associated with survival of neonates who underwent colistin therapy at a tertiary care hospital in Karachi (N=153)

\begin{tabular}{|c|c|c|c|c|}
\hline Variables & Survived $(n=111)(\%)$ & Died $(n=42)(\%)$ & OR $(95 \% \mathrm{Cl})$ & Adjusted OR $(95 \% \mathrm{CI})$ \\
\hline Mean gestational age (weeks) ${ }^{*}$ & $34.3(3.6)$ & $28.3(2.8)$ & $1.6(1.4$ to 1.9$)$ & $1.9(1.5$ to 3.0$)$ \\
\hline Mean birth weight $(\mathrm{kg})^{*}$ & $2.1(0.78)$ & $1.3(0.61)$ & $4.9(2.6$ to 9.2$)$ & $5.4(3.3$ to 11.8$)$ \\
\hline Antenatal steroids (two doses) & $33(29.7)$ & $8(19.0)$ & $1.6(0.6$ to 4.3$)$ & $1.1(0.1$ to 2.3$)$ \\
\hline History of pre-eclampsia & $71(64.0)$ & $24(57.1)$ & $1.9(0.4$ to 2.0$)$ & - \\
\hline Gestational diabetes mellitus & $22(19.8)$ & $9(21.4)$ & $4.3(0.5$ to 41.4$)$ & - \\
\hline \multicolumn{5}{|l|}{ Absence of deteriorating signs } \\
\hline Poor perfusion (inotropic support) & $60(54.1)$ & $40(95.2)$ & 17.0 (3.9 to 73.8$)$ & $6.1(2.2$ to 25.1$)$ \\
\hline Anaemia (transfusion $48-72$ hours) & $45(40.5)$ & $32(76.2)$ & 4.7 (2.1 to 10.5$)$ & 3.7 (1.9 to 7.9$)$ \\
\hline Metabolic acidosis & $29(26.1)$ & $27(64.3)$ & $5.1(2.4$ to 10.9$)$ & $4.4(2.0$ to 9.0$)$ \\
\hline Low platelets & $18(16.2)$ & $31(73.8)$ & 14.6 (6.2 to 34.2$)$ & 4.8 (2.0 to 9.2$)$ \\
\hline Ventilator requirement & $60(54.1)$ & $34(81.0)$ & 3.1 (1.5 to 8.5$)$ & $1.8(0.9$ to 4.6$)$ \\
\hline Culture grew colistin-sensitive GNB & $64(57.7)$ & $20(47.6)$ & $2.4(1.7$ to 2.9$)$ & $3.2(2.8$ to 4.0$)$ \\
\hline Infection onset (day of admission)* & $4.5(4.6)$ & $2.9(1.9)$ & $1.1(1.0$ to 1.3$)$ & $4.3(2.0$ to 9.0$)$ \\
\hline Colistin initiation after sepsis (days)* & $3.2(2.8)$ & $8.0(6.0)$ & $5.3(2.7$ to 10.8$)$ & 7.2 (3.5 to 13.6$)$ \\
\hline Achieved microbiological clearancet & $76(82.6)$ & $16(57.1)$ & $4.8(1.9$ to 11.9$)$ & $5.2(2.2$ to 13.2$)$ \\
\hline Microbial clearance (days)* & $3.6(2.3)$ & $6.0(3.1)$ & 1.7 (1.2 to 2.4$)$ & $2.0(1.9$ to 3.0$)$ \\
\hline
\end{tabular}

${ }^{*}$ Mean \pm SD.

$t n=120$ for this variable as 33 had no growth in culture.

GNB, Gram-negative bacteria. 
Table 3Table 3: Factors associated with microbial clearance among neonates suffering from sepsis who underwent colistin therapy at a tertiary care hospital in Karachi $(\mathrm{N}=120)$

\begin{tabular}{|c|c|c|c|c|}
\hline Variables & $\begin{array}{l}\text { Clearance achieved } \\
(n=92)(\%)\end{array}$ & $\begin{array}{l}\text { Clearance not achieved } \\
(n=28)(\%)\end{array}$ & $\begin{array}{l}\text { Odds Ratio } \\
(95 \% \mathrm{Cl})\end{array}$ & $\begin{array}{l}\text { Adjusted Odds Ratio } \\
(95 \% \mathrm{Cl})\end{array}$ \\
\hline Mean Gestational age (weeks)* & $33.7(3.9)$ & $30.4(4.5)$ & $1.2(1.1$ to 1.4$)$ & $1.0(0.9$ to 1.2$)$ \\
\hline Mean birth weight $(\mathrm{Kg})^{*}$ & $2.1(0.8)$ & $1.5(0.8)$ & $2.5(1.3$ to 4.6$)$ & 2.1 (1.2 to 4.2$)$ \\
\hline \multicolumn{5}{|l|}{ Absence of deteriorating signs } \\
\hline Poor perfusion (inotropic support) & $54(58.7)$ & $21(75.0)$ & 2.1 (0.8 to 5.5$)$ & 1.1 (0.4 to 3.6) \\
\hline Anemia (transfusion 48 to $72 \mathrm{hrs}$ ) & $38(41.3)$ & $20(71.4)$ & $3.6(1.5$ to 8.9$)$ & 2.2 (0.8 to 5.6$)$ \\
\hline Metabolic acidosis & $25(27.2)$ & $12(42.9)$ & $2.0(0.8$ to 4.8$)$ & $1.3(0.6$ to 3.0$)$ \\
\hline Low platelets & $23(25.0)$ & $15(53.6)$ & $3.5(1.4$ to 8.3$)$ & 2.1 (0.8 to 4.7$)$ \\
\hline Ventilator requirement & $56(60.9)$ & $18(64.3)$ & $1.1(0.5$ to 2.8$)$ & $0.8(0.4$ to 1.2$)$ \\
\hline Duration of colistin therapy (days) ${ }^{*}$ & $9.6(6.2)$ & $5.8(5.7)$ & 1.1 (1.0 to 1.2$)$ & $1.4(1.2$ to 1.6$)$ \\
\hline Culture grew colistin sensitive GNB & $66(71.7)$ & $18(64.3)$ & $1.8(0.4$ to 3.0$)$ & $2.1(1.0$ to 3.7$)$ \\
\hline Infection onset (day of admission)* & $4.9(4.8)$ & $2.9(2.3)$ & $1.2(1.0$ to 1.4$)$ & $2.0(1.0$ to 1.5$)$ \\
\hline Colistin initiation after sepsis (days)* & $2.3(3.3)$ & $5.4(6.1)$ & $2.9(1.8$ to 3.3$)$ & $3.9(2.3$ to. 4.8$)$ \\
\hline Bacteraemia & $82(89.1)$ & $25(89.3)$ & $1.0(0.3$ to 3.9$)$ & \\
\hline Pneumonia & $20(21.7)$ & $9(32.1)$ & $0.6(0.2$ to 1.5$)$ & \\
\hline Meningitis & $6(6.5)$ & $9(32.1)$ & $0.9(0.2$ to 3.6$)$ & \\
\hline \multicolumn{5}{|l|}{ Isolated microorganisms } \\
\hline Acinetobacter baumannii & $33(35.9)$ & $10(35.7)$ & $1.0(0.4$ to 2.4$)$ & \\
\hline Klebsiella pneumonia & $34(37.0)$ & $8(28.6)$ & $1.5(0.6$ to 3.7$)$ & \\
\hline Enterobacter species & $11(12.0)$ & $1(3.6)$ & $3.7(0.5$ to 30.0$)$ & \\
\hline Pseudomonas aeruginosa & $8(8.7)$ & $5(17.9)$ & $0.4(0.1$ to 1.5$)$ & \\
\hline Colistin administered within 1-2hrs of severe sepsis/septic shock & $82(89.1)$ & $4(14.3)$ & $49.2(14.2$ to 17.9$)$ & $32.9(15.4$ to 58.5$)$ \\
\hline Simultaneous use of carbapenems & $83(90.2)$ & $26(92.9)$ & 0.7 (0.1 to 3.5$)$ & \\
\hline
\end{tabular}

${ }^{*}$ Mean \pm SD.

GNB, Gram-negative bacteria.

microbial clearance. However, the absence of deteriorating signs and GA had no association with microbial clearance.

\section{DISCUSSION}

Despite the reintroduction of colistin and its wide use among adults for MDR-GNB infections, there is very little evidence of colistin use in neonates. Few studies have reported intravenous use of colistin. ${ }^{273031}$ This is one of the few studies with a reasonable sample size to determine the effectiveness and adverse effects of colistin in the critically ill neonatal population. Our study found that colistin use was associated with $72.5 \%$ survival among neonates with sepsis. Alan et al reported 4/21 deaths in neonates treated with colistin; however, their study was limited in size and deaths within 72 hours of birth were not included. ${ }^{18}$ Serafettin Tekgunduz et al reported a 50\% mortality among neonates with culture-proven sepsis treated with colistin. ${ }^{31}$

The study also evaluated the predictors of survival and microbial clearance among neonates given colistin therapy. As expected, small for GA and low birthweight babies had higher mortality. These findings are consistent with several other studies globally. ${ }^{27} 32$ The absence of deteriorating clinical signs and laboratory parameters was significantly associated with survival, findings which are consistent and biologically plausible. ${ }^{33}$ Nonetheless, the duration of colistin therapy, the type of organism treated and the site of infection had no association with survival and microbial clearance. On the other hand, the presence of colistin-sensitive GNB and early initiation of colistin therapy after the onset of sepsis (especially administration of first intravenous dose within 1-2 hours of the onset of severe sepsis/septic shock) were associated with increased survival of the neonates and microbial clearance. To the best of our knowledge, this is the first study of its kind which has looked at the association of these factors in a neonatal population.

The concomitant use of other antimicrobial agents had no association with survival or microbial clearance. Studies have shown that long-term use of colistin leads to microbial resistance; however, the duration of therapy was short in our study and none showed subsequent resistance. ${ }^{34}$

To the best of our knowledge, this is the first study to report the use of intrathecal colistin for meningitis in neonates. In our study, 15 patients had meningitis, 7 received intrathecal colistin (5 of whom also received intravenous colistin); the other 8 patients with meningitis received only intravenous colistin. All neonates with meningitis who received both intravenous and intrathecal colistin survived, but none of those given intravenous colistin alone survived. Furthermore, two neonates who received only intrathecal colistin; one survived and one died. This suggests that the appropriate route of colistin has a significant impact on clinical outcomes, especially in neonates with ventriculitis and meningitis with colistin-sensitive GNB. However, these results should be interpreted with caution, as there was a small number of neonates in these categories. We recommend further studies in this regard.

Nephrotoxicity is the major side effect related to colistin use reported in the literature. ${ }^{9}$ Though we followed the definition for AKI used in previous neonatal studies, the rate of nephrotoxicity was much lower in our study. ${ }^{85-37}$ A study of very low birth weight (VLBW) neonates given a similar dose of colistin $(5 \mathrm{mg} / \mathrm{kg} / \mathrm{day})^{27}$ found AKI in 4/28 (14\%) VLBW neonates. This might be related to a shorter duration of colistin in our study compared with other studies with a higher prevalence of AKI. ${ }^{893839}$ Bartter-like syndrome was reported in a neonate with 
26 days of therapy ${ }^{18}$ In our study, colistin was continued in AKI patients with dose adjustment according to weight and serum creatinine. The concomitant use of nephrotoxic medications did not seem to affect AKI development.

Overall, 28 (18.3\%) patients in our study experienced reversible hyponatraemia, hypokalaemia, hypomagnesaemia and hypocalcaemia. These adverse effects were lower (16.2\%) among patients who survived than those who died (23.8\%). Colistin therapy was not discontinued in these cases who were managed with supportive care. ${ }^{1840}$ The need for supplemental electrolytes in our study was lower than the previous VLBW neonatal study, which reported $78.6 \%$ and $53.6 \%$ neonates needing magnesium and potassium supplementations, respectively. ${ }^{27}$

In the present study, seizures were observed in 14 (12.6\%) who survived and $7(16.7 \%)$ of those who died. ${ }^{8}$ Of these 21 neonates, 3 had meningomyelocele, 1 each had brainstem bleed, an external ventricular drain/ventriculoperitoneal shunt, 5 had perinatal asphyxia, 2 had hydrocephalus, 4 had intraventricular haemorrhage (IVH), and 3 had IVH and hydrocephalus. A total of $8 / 21$ had meningitis. It was not possible to directly correlate the neurological side effects with colistin therapy, given the comorbidities, clinical circumstances and other medications in these sick neonates. ${ }^{818}$ Overall, the number of patients in each category was too small to deduce any meaningful conclusions.

Our study is the largest reported study of neonatal colistin use, and we found successful colistin therapy via inhalation, intrathecal and intravenous routes with tolerable adverse effects. A few limitations must be considered. This was a single-centre retrospectively designed study. Also, some of the positive isolates in urine and tracheal aspirate could be colonising species rather than causative pathogens. Further prospective studies need to be conducted to confirm our findings.

In conclusion, this study suggests that colistin may be safe and effective for treating MDR-GNB infections in critically ill neonates. However, it remains the last resort for MDR-GNB after the failure of carbapenems. Moreover, for the optimal neonatal doses through different routes of administration, further pharmacodynamics and pharmacokinetic studies are required.

Correction notice This paper has been corrected since it was published online. There was a data error in table 1 in the row 'Diagnosis/site of infection', for meningitis and pneumonia.

Acknowledgements We would like to acknowledge the technical and editing assistance by Abdul Moiz Hussain and Dr Imran Nisar.

Funding The authors have not declared a specific grant for this research from any funding agency in the public, commercial or not-for-profit sectors.

Competing interests None declared.

Patient consent for publication Not required.

Ethics approval Ethical approval was obtained before starting this study from the Ethical Review Committee (ERC) of Aga Khan University, Karachi, Pakistan (ERC \# 2018-0233-137)

Provenance and peer review Not commissioned; externally peer reviewed.

Data availability statement Data are available upon reasonable request.

ORCID iD

Gul Ambreen http://orcid.org/0000-0002-6287-6898

\section{REFERENCES}

1 Souli M, Galani I, Giamarellou H. Emergence of extensively drug-resistant and pandrug-resistant gram-negative bacilli in Europe. Euro Surveill 2008;13:19045.

2 Berlana D, Llop JM, Fort E, et al. Use of colistin in the treatment of multiple-drugresistant gram-negative infections. Am J Health Syst Pharm 2005;62:39-47.

3 Karaiskos I, Galani L, Baziaka F, et al. Intraventricular and intrathecal colistin as the last therapeutic resort for the treatment of multidrug-resistant and extensively drugresistant Acinetobacter baumannii ventriculitis and meningitis: a literature review. Int J Antimicrob Agents 2013;41:499-508.
4 Saleem AF, Ahmed I, Mir F, et al. Pan-resistant Acinetobacter infection in neonates in Karachi, Pakistan. The Journal of Infection in Developing Countries 2010;4:030-7.

5 Saleem AF, Qamar FN, Shahzad H, et al. Trends in antibiotic susceptibility and incidence of late-onset Klebsiella pneumoniae neonatal sepsis over a six-year period in a neonatal intensive care unit in Karachi, Pakistan. Int J Infect Dis 2013;17:e961-5.

6 Investigators of the Delhi Neonatal Infection Study (DeNIS) collaboration. Characterisation and antimicrobial resistance of sepsis pathogens in neonates born in tertiary care centres in Delhi, India: a cohort study. Lancet Glob Health 2016:4:e752-60

7 Ozsurekci Y, Aykac K, Cengiz AB, et al. Bloodstream infections in children caused by carbapenem-resistant versus carbapenem-susceptible gram-negative microorganisms: risk factors and outcome. Diagn Microbiol Infect Dis 2017:87:359-64.

8 Nation RL, Li J. Colistin in the 21st century. Curr Opin Infect Dis 2009;22:535-43.

9 Biswas S, Brunel J-M, Dubus J-C, et al. Colistin: an update on the antibiotic of the 21st century. Expert Rev Anti Infect Ther 2012;10:917-34.

10 Kazmierczak KM, Tsuji M, Wise MG, et al. In vitro activity of cefiderocol, a siderophore cephalosporin, against a recent collection of clinically relevant carbapenem-nonsusceptible gram-negative bacilli, including serine carbapenemase- and metallo$\beta$-lactamase-producing isolates (SIDERO-WT-2014 study). Int J Antimicrob Agents 2019;53:177-84.

11 Dobias J, Dénervaud-Tendon V, Poirel L, et al. Activity of the novel siderophore cephalosporin cefiderocol against multidrug-resistant gram-negative pathogens. Eur J Clin Microbiol Infect Dis 2017;36:2319-27.

12 Thomas R, Velaphi $S$, Ellis $S$, et al. The use of polymyxins to treat carbapenem resistant infections in neonates and children. Expert Opin Pharmacother 2019;20:415-22.

13 Koch-Weser J, Sidel VW, Federman EB, et al. Adverse effects of sodium colistimethate. manifestations and specific reaction rates during 317 courses of therapy. Ann Intern Med 1970;72:857-68.

14 Ramasubban S, Majumdar A, Das PS. Safety and efficacy of polymyxin B in multidrug resistant gram-negative severe sepsis and septic shock. Indian J Crit Care Med 2008;12:153-7.

15 Durakovic N, Radojcic V, Boban A, et al. Efficacy and safety of colistin in the treatment of infections caused by multidrug-resistant Pseudomonas aeruginosa in patients with hematologic malignancy: a matched pair analysis. Intern Med 2011;50:1009-13.

16 Al-Lawama M, Aljbour H, Tanash A, et al. Intravenous colistin in the treatment of multidrug-resistant Acinetobacter in neonates. Ann Clin Microbiol Antimicrob 2016;15:8.

17 Jajoo M, Kumar V, Jain M, et al. Intravenous colistin administration in neonates. Pediatr Infect Dis J 2011;30:218-21.

18 Alan S, Yildiz D, Erdeve 0, et al. Efficacy and safety of intravenous colistin in preterm infants with nosocomial sepsis caused by Acinetobacter baumannii. Am J Perinatol 2014;31:1079-86.

19 Horan TC, Andrus M, Dudeck MA. CDC/NHSN surveillance definition of health care-associated infection and criteria for specific types of infections in the acute care setting. Am J Infect Control 2008;36:309-32.

20 Kamath BD, Macguire ER, McClure EM, et al. Neonatal mortality from respiratory distress syndrome: lessons for low-resource countries. Pediatrics 2011;127:1139-46.

21 Jetton JG, Askenazi DJ. Update on acute kidney injury in the neonate. Curr Opin Pediatr 2012;24:191-6.

22 Magiorakos A-P, Srinivasan A, Carey RB, et al. Multidrug-Resistant, extensively drugresistant and pandrug-resistant bacteria: an international expert proposal for interim standard definitions for acquired resistance. Clin Microbiol Infect 2012;18:268-81.

23 Swaminathan R. Magnesium metabolism and its disorders. Clin Biochem Rev 2003;24:47

24 Dhondup T, Qian Q. Acid-Base and electrolyte disorders in patients with and without chronic kidney disease: an update. Kidney Dis 2017;3:136-48

25 Wayne P. Clinical and laboratory Standards Institute. performance standards for antimicrobial susceptibility testing. 2011

26 Mok K, Christian MD, Nelson S, et al. Time to administration of antibiotics among inpatients with severe sepsis or septic shock. Can J Hosp Pharm 2014;67:213.

27 Ilhan 0 , Bor M, Ozdemir SA, et al. Efficacy and safety of intravenous colistin in very low birth weight preterm infants. Paediatr Drugs 2018;20:475-81.

28 Nakwan N, Wannaro J, Thongmak T, et al. Safety in treatment of ventilator-associated pneumonia due to extensive drug-resistant Acinetobacter baumannii with aerosolized colistin in neonates: a preliminary report. Pediatr Pulmonol 2011;46:60-6.

29 Saleem AF, Shah MS, Shaikh AS, et al. Acinetobacter species meningitis in children: a case series from Karachi, Pakistan. J Infect Dev Ctries 2011;5:809-14.

30 Antachopoulos C, losifidis E. Colistin use in neonates and children with infections due to carbapenem-resistant bacteria. Pediatr Infect Dis J 2017;36:905-7.

31 Serafettin Tekgunduz K, Kara M, Caner I, et al. Safety and efficacy of intravenous colistin in neonates with culture proven sepsis. Iran J Pediatr 2015;25:e453.

32 Jasani B, Kannan S, Nanavati $R$, et al. An audit of colistin use in neonatal sepsis from a tertiary care centre of a resource-limited country. Indian J Med Res 2016;144:433.

33 Huang Y-C, Su L-H, Wu T-L, et al. Outbreak of Acinetobacter baumannii bacteremia in a neonatal intensive care unit: clinical implications and genotyping analysis. Pediatr Infect Dis J 2002;21:1105-9.

34 Çağan E, Kıray Baş E, Asker HS. Use of colistin in a neonatal intensive care unit: a cohort study of 65 patients. Med Sci Monit 2017;23:548-54. 


\section{Original research}

35 Celik IH, Demirel G, Tatar Aksoy H, et al. [Acinetobacter baumannii: an important pathogen with multidrug resistance in newborns]. Mikrobiyol Bul 2011;45:716-22.

36 Alaoui SY, Nejmi SE, Chakir AA, et al. [Intraventricular colistin use in neonatal meningitis caused by Acinetobacter baumanii]. Ann Fr Anesth Reanim 2011;30:8545 .

37 Karbuz A, Özdemir H, Yaman A, et al. The use of colistin in critically ill children in a pediatric intensive care unit. Pediatr Infect Dis J 2014;33:e19-24.
38 Iosifidis E, Antachopoulos C, loannidou M, et al. Colistin administration to pediatric and neonatal patients. Eur J Pediatr 2010;169:867-74.

39 Hartzell JD, Neff R, Ake J, et al. Nephrotoxicity associated with intravenous colistin (colistimethate sodium) treatment at a tertiary care medical center. Clin Infect Dis 2009;48:1724-8

40 Cakir U, Alan S, Zeybek C, et al. Acquired Bartter-like syndrome associated with colistin use in a preterm infant. Ren Fail 2013;35:411-3. 